\title{
Parametric excitation in a two degree of freedom MEMS system ${ }^{1}$
}

\author{
Johannes Welte ${ }^{\mathrm{a}, *}$, Till Jochen Kniffka ${ }^{\mathrm{b}}$ and Horst Ecker ${ }^{\mathrm{b}}$ \\ ${ }^{a}$ Institute for Transport Studies, University of Natural Resources and Life Sciences, Vienna, Austria \\ ${ }^{\mathrm{b}}$ Institute of Mechanics and Mechatronics, Vienna University of Technology, Vienna, Austria
}

\begin{abstract}
This contribution investigates the influence of parametric excitation on the dynamic stability of a microelectromechanical system. In systems with just a single degree of freedom, parametric excitation causes the oscillator to exhibit unstable behavior within certain intervals of the parametric excitation frequency. In multi-degree of freedom systems on the other hand, unstable behavior is caused within a wider range of intervals of the parametric excitation frequency. Moreover, such systems show frequency intervals of enhanced stability, an effect known as anti-resonance phenomenon. Both types of phenomena, the parametric resonance and anti-resonance, are modeled and studied for a microelectromechanical system with two degrees of freedom and some novel results are discussed.
\end{abstract}

Keywords: Stability, resonance, anti-resonance, parametric excitation, MEMS

\section{Introduction}

Microelectromechanical systems (MEMS) are becoming more and more important for all kinds of industrial applications. The global market for micro-electromechanical systems, which includes products such as sensors in automobile airbag systems, actuators in printer cartridges and many others reached 40 billion USD already in 2006, according to economic sources. Therefore, there is a huge market for new developments, but also for improvements of already existing technology.

Due to the many difficulties in the production process [8], MEMS consist of rather simple mechanical structures [6]. In general the number of independent motions of such a system is very limited. As a result it is not a surprise that single degree of freedom (1-dof) systems prevail for the most part in present industrial applications. Although the design has to be rather simple from a mechanical point of view, the electro-mechanical properties of such micro-devices are frequently more complicated than equivalent systems in the macro-world. Especially the presence of non-linear characteristics, the almost complete absence of external damping and other factors contribute to the complex behavior of such low-dimensional MEMS. On the other hand, the combination of electrical and mechanical forces in such devices make it possible to take advantage of physical effects that are not useful, occasionally even strictly avoided, in other applications.

One of such ideas is the introduction of time-periodic parameters to a microelectromechanical system. In one of the first papers [7] on this topic, Turner et al. proposed to operate a single degree of freedom (1-dof) MEMS oscillators at a so-called parametric resonance instead of a conventional resonance. The advantage of this concept lies in the fundamental difference between an ordinary resonance phenomenon and a parametric resonance. The latter destabilizes the system and causes the oscillation amplitudes to grow exponentially with time, rather than

\footnotetext{
${ }^{1}$ Paper has been selected for publication in: Special Issue on Nonlinear Vibrations in Elastic Structures: Dynamics and Control. Editors Balthazar, Batista and Fenili (ICNPAA 2012, Vienna, Austria).

${ }^{*}$ Corresponding author: Johannes Welte, Institute for Transport Studies, University of Natural Resources and Life Sciences, Gregor Mendel Strã̃ße 33, 1180 Vienna, Austria. E-mail: johannes.welte@boku.ac.at.
} 


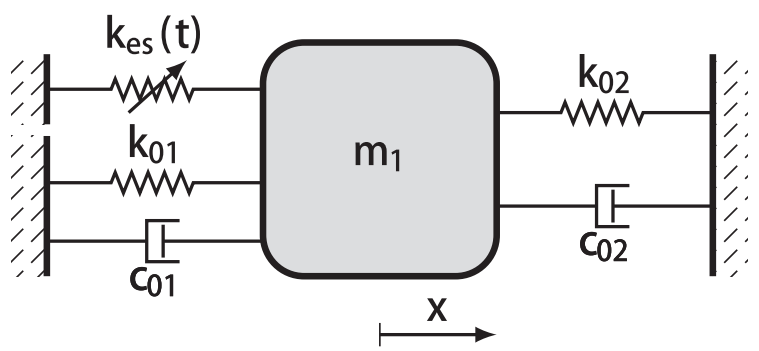

Fig. 1. Mechanical model of the 1-dof MEMS oscillator.

linear as for the conventional system. Since a fast response and growth of a signal is frequently the desired effect, such parametrically excited MEMS exhibit an improved performance compared to a conventional design, see [10] for example.

Due to the possibility of tuning parametrically excited MEMS oscillators, as discussed in [1,11], the frequency ranges where parametric resonances might occur can be adjusted. This makes it possible to significantly increase the accuracy of mass sensors as presented, for example, in $[14,16,18,20]$. The mass sensing oscillator is thereby deliberately operated at a parametric resonance and makes use of the fast transition between a stable and an unstable state of motion.

Another possible application is to use a MEMS as a parametric amplifier, see [5]. This offers several advantages compared to a semiconductor based design, as it is more heat resistant and has generally lower signal noise. The filtering of electronic signals is yet another field of application. Due to the growing demand for efficient and accurate filtering of signals, parametrically excited MEMS can help to further improve the systems quality. Basic MEMS filter designs and detailed investigations are presented in $[9,12]$ for example.

While parametric excitation (PE) is used so far to take advantage of parametric resonances, this contribution suggests to also exploit so-called parametric anti-resonances in order to improve the damping behavior of such systems. As vibration damping is typically low in microelectromechanical system since they operate in vacuum, there is a need for adjusting and amplifying the damping properties of such systems. As the parametric anti-resonance phenomenon requires a system with at least two degrees of freedom, modeling aspects of a 2-dof MEM system and some first results are the focus of this paper.

Section 2 starts off with a brief review of a parametrically excited 1-dof MEMS oscillator as proposed and discussed in the recent literature. In Section 3 the MEMS is expanded by an additional degree of freedom, enabling the system to not only extend its parametric regions of instability (see Section 4), but also to exploit parametric anti-resonances. This specific vibration phenomenon is discussed in Section 5. The paper concludes in Section 6, summarizing the present work.

\section{Single degree of freedom MEMS oscillator}

A typical single degree of freedom MEMS oscillator may consist of a backbone with two elastic beam springs connected on each side (see [8]). In order to force the system into motion, a so-called comb drive may be connected to the backbone. Typical comb drives are operated by alternating voltage signals, resulting in a time dependent mechanical stiffness acting on the backbone. Such a MEMS oscillator can be modeled by a mechanical spring-mass system as shown in Fig. 1.

The corresponding differential equation can be written as

$$
m \ddot{x}+\left(c_{01}+c_{02}\right) \dot{x}+\left(k_{01}^{l i n}+k_{02}^{l i n}\right) x+k_{e s}^{l i n}(t) x+\left(k_{01}^{n l i n}+k_{02}^{n l i n}\right) x^{3}+k_{e s}^{n l i n}(t) x^{3}=0,
$$

with $x$ being the system's deflection. The linear damping coefficients are denoted $c_{01}$ and $c_{02}$, whereas the stiffness coefficients of the elastic beams are denoted $k_{01}$ and $k_{02}$. The nonlinear mechanical stiffness provided by the beams is accounted for by nonlinear stiffness coefficients multiplied by cubic terms of the deflection. According to [10] the electrostatic stiffness provided by the comb drive can be modeled by

$$
k_{e s}(t)=\left(r_{1 A} x+r_{3 A} x^{3}\right) V_{A}^{2}[1+\cos (\omega t)]
$$


Table 1

Nondimensional parameter definitions adapted from [9]

\begin{tabular}{ll}
\hline Definition & Nondimensional parameter \\
\hline$z^{\prime}=\frac{d z}{d \tau}$ & Scaled time derivative of deflection \\
$\Omega=\frac{\omega}{\hat{\omega}}$ & Nondimensional parametric excitation frequency \\
$\eta_{i}=\frac{\omega}{\hat{\omega}}$ & Nondimensional natural frequency \\
$\xi_{1}=\frac{c_{01}}{2 m \hat{\omega}}$ & Scaled damping ratio for $c_{01}$ \\
$\xi_{2}=\frac{c_{02}}{2 m \hat{\omega}}$ & Scaled damping ratio for $c_{02}$ \\
$\lambda_{1}=\frac{r_{1 A} V_{A}^{2}}{k_{01}^{l i n}+k_{02}^{l i n}}$ & Linear electrostatic excitation amplitude \\
$\lambda_{3}=\frac{x_{0}^{2} r_{3 A} V_{A}^{2}}{k_{01}^{l i n}+k_{02}^{l i n}}$ & Nonlinear electrostatic excitation coefficient \\
$\chi=\frac{\left(k_{01}^{n l i n}+k_{02}^{n l i n}\right.}{k_{01}^{l i n}+k_{02}^{l i n}} x_{0}^{2}$ & Nonlinear mechanical stiffness coefficient \\
\hline
\end{tabular}

where $r_{1 A}$ and $r_{3 A}$ are the linear and nonlinear electrostatic stiffness coefficients, influenced by the particular design of the comb drive (see [19]). The amplitude of the alternating voltage input signal is denoted $V_{A}$. To make the differential equation nondimensional, a dimensionless parametric excitation frequency $\Omega$ is introduced. This frequency is related to the actual parametric excitation frequency $\omega$ as follows

$$
\Omega=\frac{\omega}{\hat{\omega}}
$$

where $\hat{\omega}$ is a characteristic reference frequency. In the present case, given a system with just a single degree of freedom, it is beneficial to use the natural frequency of the linearized system $\hat{\omega}$ as a reference. In order to obtain a scaled system time, a dimensionless time $\tau$ is introduced, which leads to

$$
\tau=\hat{\omega} t, \quad \omega t=\Omega \hat{\omega} t=\Omega \tau .
$$

The deflection $x$ of the single backbone mass is rescaled according to

$$
z=\frac{x}{x_{0}}
$$

where $x_{0}$ is a scaling parameter of suitable size related to the physical dimensions of the MEMS. Carrying out the rescaling leads to a nondimensional differential equation of the form

$$
z^{\prime \prime}+2\left(\xi_{1}+\xi_{2}\right) z^{\prime}+\left(1+\lambda_{1}\right) z+\left[\cos (\Omega \tau) \lambda_{1}\right] z+\left(\chi+\lambda_{3}\right) z^{3}+\left[\cos (\Omega \tau) \lambda_{3}\right] z^{3}=0
$$

where the newly introduced differential operators and the linear and nonlinear nondimensional parameters are defined as stated in Table 1.

It is widely known that single degree of freedom parametrically excited systems exhibit parametric resonances at excitation frequencies of

$$
\Omega_{k}=\frac{2 \eta_{1}}{k}, \quad k=1,2,3, \ldots
$$

where $\eta_{1}$ is the normalized natural frequency of the system. In recent publications (e.g. [10] or [19]), the primary parametric resonance $2 \eta_{1}$ has been investigated for systems similar to the one shown in Fig. 1. Due to presence of damping the parametric resonances of higher order $(k>1)$ are of limited practical interest.

As a possible application, it is suggested to use parametrically excited MEMS oscillators for electronic filtering. Because of the sharp transition between stable and unstable system behavior, the quality of a filter may be improved significantly. Keeping that application in mind, a MEMS with two degrees of freedom is modeled and analyzed. 


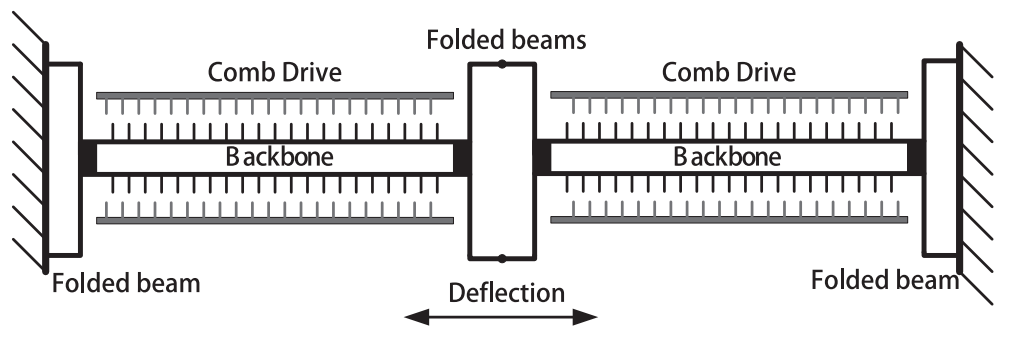

Fig. 2. Basic design of the 2-dof MEMS.

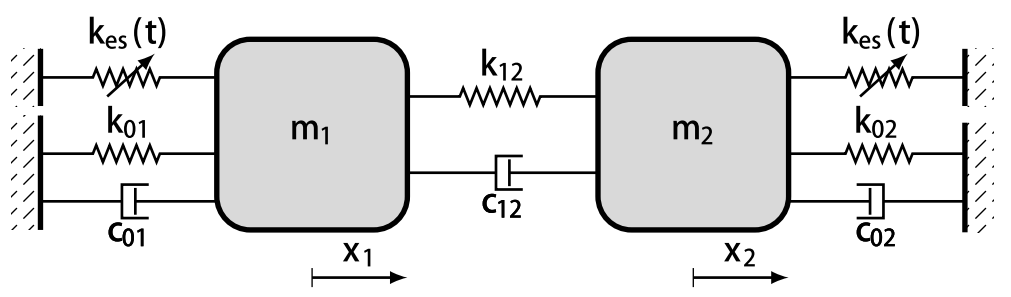

Fig. 3. Mechanical model of the 2-dof MEMS oscillator

\section{MEMS oscillator with two degrees of freedom}

The MEMS oscillator with two degrees of freedom is based on the single degree of freedom design and supplemented by an additional backbone, spring and comb drive. The basic structure of the resulting oscillator is shown in Fig. 2.

\subsection{Modeling the 2-dof MEMS oscillator}

The mechanical model of the two degree of freedom MEMS oscillator is depicted in Fig. 3. It can be seen that due to the second comb drive, a time dependent electrostatic stiffness is present on both sides of the oscillator.

The differential equations in matrix form is derived as follows

$$
\mathbf{M} \ddot{\mathbf{x}}+\mathbf{C} \dot{\mathbf{x}}+\mathbf{K}^{l i n} \mathbf{x}+\mathbf{K}^{n l i n} \mathbf{x}^{3}+\cos (\omega t) \mathbf{P}_{C}^{l i n} \mathbf{x}+\cos (\omega t) \mathbf{P}_{C}^{n l i n} \mathbf{x}^{3}=\mathbf{0},
$$

with the vector of deflections given by

$$
\mathbf{x}=\left[x_{1} x_{2}\right]^{T}
$$

The mass matrix $\mathbf{M}$ and the damping matrix $\mathbf{C}$ are derived as follows

$$
\begin{aligned}
& \mathbf{M}=\left[\begin{array}{cc}
m_{1} & 0 \\
0 & m_{2},
\end{array}\right] \\
& \mathbf{C}=\left[\begin{array}{cc}
c_{01}+c_{12} & -c_{12} \\
-c_{12} & c_{02}+c_{12}
\end{array}\right] .
\end{aligned}
$$

The mechanical stiffness matrix $\mathbf{K}$ may be split up in a linear and a nonlinear part. By introducing the following stiffness coefficients

$$
\begin{gathered}
\alpha^{l i n}=k_{12}^{l i n}+r_{1 A} V_{A}^{2}, \\
\alpha^{n l i n}=k_{12}^{n l i n}+r_{3 A} V_{A}^{2}
\end{gathered}
$$


Table 2

Nondimensional parameter definitions for the 2-dof MEMS oscillator

\begin{tabular}{ll}
\hline Definition & Nondimensional parameter \\
\hline$z_{1,2}^{\prime}=\frac{d z_{1,2}}{d \tau}$ & Scaled time derivative of deflections \\
$\Omega=\frac{\omega}{\hat{\omega}}$ & Nondimensional parametric excitation frequency \\
$\gamma=\frac{m_{1}}{m_{2}}$ & Mass ratio \\
$\xi_{01}=\frac{c_{01}}{2 m_{1} \hat{\omega}}$ & Scaled damping ratio for $c_{01}$ \\
$\xi_{12}=\frac{c_{12}}{2 m_{1} \hat{\omega}}$ & Scaled damping ratio for $c_{12}$ \\
$\xi_{02}=\frac{c_{02}}{2 m_{1} \hat{\omega}}$ & Scaled damping ratio for $c_{02}$ \\
$\lambda_{1}=\frac{r_{1 A} V_{A}^{2}}{k_{01}^{\operatorname{lin}}}$ & Linear electrostatic excitation amplitude \\
$\lambda_{3}=\frac{x_{0}^{2} r_{3 A} V_{A}^{2}}{k_{01}^{l i n}}$ & Nonlinear electrostatic excitation coefficient \\
$\beta_{12}=\frac{k_{12}^{l i n}}{k_{01}^{\operatorname{lin}}}$ & Linear mechanical stiffness quotient \\
$\beta_{02}=\frac{k_{02}^{l i n}}{k_{01}^{l i n}}$ & Linear mechanical stiffness quotient \\
$\chi_{01}=\frac{k_{01}^{n l i n} x_{0}^{2}}{k_{01}^{\operatorname{lin}}}$ & Nonlinear mechanical stiffness coefficient \\
$\chi_{12}=\frac{k_{12}^{n l i n} x_{0}^{2}}{k_{01}^{\operatorname{lin}}}$ & Nonlinear mechanical stiffness coefficient \\
$\chi_{02}=\frac{k_{02}^{n l i n} x_{0}^{2}}{k_{01}^{l i n}}$ & Nonlinear mechanical stiffness coefficient \\
\hline
\end{tabular}

one obtains the linear and nonlinear stiffness matrix

$$
\begin{aligned}
& \mathbf{K}^{l i n}=\left[\begin{array}{cc}
k_{01}^{l i n}+\alpha^{l i n} & -k_{12}^{l i n} \\
-k_{12}^{l i n} & k_{02}^{l i n}+\alpha^{l i n}
\end{array}\right], \\
& \mathbf{K}^{n l i n}=\left[\begin{array}{cc}
k_{01}^{n l i n}+\alpha^{n l i n}+3 k_{12}^{n l i n} \frac{x_{2}^{2}}{x_{1}^{2}} & -k_{12}^{n l i n}-3 k_{12}^{n l i n} \frac{x_{1}^{2}}{x_{2}^{2}} \\
-k_{12}^{n l i n}-3 k_{12}^{n l i n} \frac{x_{2}^{2}}{x_{1}^{2}} & k_{02}^{n l i n}+\alpha^{n l i n}+3 k_{12}^{n l i n} \frac{x_{1}^{2}}{x_{2}^{2}}
\end{array}\right] .
\end{aligned}
$$

The electrostatic stiffness can be expressed by Eq. (2), resulting in the linear and nonlinear parametric excitation matrix

$$
\begin{aligned}
& \mathbf{P}_{C}^{l i n}=\left[\begin{array}{cc}
r_{1 A} V_{A}^{2} & 0 \\
0 & r_{1 A} V_{A}^{2}
\end{array}\right], \\
& \mathbf{P}_{C}^{n l i n}=\left[\begin{array}{cc}
r_{3 A} V_{A}^{2} & 0 \\
0 & r_{3 A} V_{A}^{2}
\end{array}\right] .
\end{aligned}
$$

For an easier interpretation of the upcoming simulation results and for efficient numerical treatment, the nonlinear differential Eq. (8) is scaled. Therefore, a nondimensional parametric excitation frequency $\Omega$ is introduced the same way as in Eq. (3), with the characteristic reference frequency $\hat{\omega}$ being defined as the natural frequency of a linearized subsystem. This subsystem can be derived from the original system shown in Fig. 3 by setting every stiffness (and damping) coefficient to zero, except for $k_{01}^{\text {lin }}$. The remaining subsystem consist only of mass $m_{1}$ and the linear mechanical spring $k_{01}^{\text {lin }}$. The natural frequency of that subsystem is known to be

$$
\hat{\omega}=\sqrt{\frac{k_{01}^{\operatorname{lin}}}{m_{1}} .}
$$

To obtain a scaled system time, a dimensionless time $\tau$ is introduced by multiplying time $t$ with the reference frequency $\hat{\omega}$ according to Eq. (4). The displacements $x_{1}$ and $x_{2}$ of the oscillator are rescaled using a convenient scaling parameter $x_{0}$

$$
z_{1}=\frac{x_{1}}{x_{0}}, \quad z_{2}=\frac{x_{2}}{x_{0}} .
$$


Table 3

Parameter values for a two degrees of freedom MEMS

\begin{tabular}{lll}
\hline Parameter & Value & Units \\
\hline$m_{1}$ & $1.8 \times 10^{-10}$ & $\mathrm{~kg}$ \\
$m_{2}$ & $3.6 \times 10^{-10}$ & $\mathrm{~kg}$ \\
$c_{01}$ & $6.0 \times 10^{-8}$ & $\mathrm{Ns} / \mathrm{m}$ \\
$c_{12}$ & $6.0 \times 10^{-8}$ & $\mathrm{Ns} / \mathrm{m}$ \\
$c_{02}$ & $4.0 \times 10^{-7}$ & $\mathrm{Ns} / \mathrm{m}$ \\
$k_{01}^{l i n}$ & 1.9 & $\mu \mathrm{N} / \mu \mathrm{m}$ \\
$k_{12}^{l i n}$ & 1.4 & $\mu \mathrm{N} / \mu \mathrm{m}$ \\
$k_{02}^{l i n}$ & 8.0 & $\mu \mathrm{N} / \mu \mathrm{m}$ \\
$k_{01}^{n l i n}$ & 0.018 & $\mu \mathrm{N} / \mu \mathrm{m}^{3}$ \\
$k_{12}^{n l i n}$ & 0.009 & $\mu \mathrm{N} / \mu \mathrm{m}^{3}$ \\
$k_{02}^{n l i n}$ & 0.018 & $\mu \mathrm{N} / \mu \mathrm{m}^{3}$ \\
$r_{1 A}$ & $3.8 \times 10^{-4}$ & $\mu \mathrm{N} / \mathrm{V}^{2} \mu \mathrm{m}$ \\
$r_{3 A}$ & $-1.6 \times 10^{-5}$ & $\mu \mathrm{N} / \mathrm{V}^{2} \mu \mathrm{m}^{3}$ \\
\hline
\end{tabular}

Carrying out the rescaling substitutions leads to the following nondimensional matrix differential equation for the unknowns $\mathbf{z}=\left[\begin{array}{ll}z_{1} & z_{2}\end{array}\right]^{T}$

$$
\mathbf{z}^{\prime \prime}+\hat{\mathbf{C}} \mathbf{z}^{\prime}+\hat{\mathbf{K}}^{l i n} \mathbf{z}+\hat{\mathbf{K}}^{n l i n} \mathbf{z}^{3}+\cos (\Omega \tau) \hat{\mathbf{P}}_{C}^{l i n} \mathbf{z}+\cos (\Omega \tau) \hat{\mathbf{P}}_{C}^{n l i n} \mathbf{z}^{3}=\mathbf{0},
$$

with the newly introduced nondimensional parameters defined as stated in Table 2.

The scaled damping matrix is defined by

$$
\hat{\mathbf{C}}=\left[\begin{array}{cc}
2\left(\xi_{01}+\xi_{12}\right) & -2 \xi_{12} \\
-2 \gamma \xi_{12} & 2 \gamma\left(\xi_{02}+\xi_{12}\right)
\end{array}\right]
$$

Defining the parameter

$$
\hat{\alpha}^{n l i n}=\chi_{12}+\lambda_{3},
$$

leads to the following scaled stiffness matrices

$$
\begin{aligned}
& \hat{\mathbf{K}}^{l i n}=\left[\begin{array}{cc}
1+\beta_{12}+\lambda_{1} & -\beta_{12} \\
-\gamma \beta_{12} & \gamma\left(\beta_{02}+\beta_{12}+\lambda_{1}\right)
\end{array}\right], \\
& \hat{\mathbf{K}}^{n l i n}=\left[\begin{array}{cc}
\chi_{01}+\hat{\alpha}^{n l i n}+3 \chi_{12} \frac{z_{2}^{2}}{z_{1}^{2}} & -\chi_{12}-3 \chi_{12} \frac{z_{1}^{2}}{z_{2}^{2}} \\
\gamma\left(-\chi_{12}-3 \chi_{12} \frac{z_{2}^{2}}{z_{1}^{2}}\right)^{2} & \gamma\left(\chi_{02}+\hat{\alpha}^{n l i n}+3 \chi_{12} \frac{z_{1}^{2}}{z_{2}^{2}}\right)
\end{array}\right]
\end{aligned}
$$

and parametric excitation matrices

$$
\begin{aligned}
& \hat{\mathbf{P}}_{C}^{l i n}=\left[\begin{array}{cc}
\lambda_{1} & 0 \\
0 & \gamma \lambda_{1}
\end{array}\right], \\
& \hat{\mathbf{P}}_{C}^{n l i n}=\left[\begin{array}{cc}
\lambda_{3} & 0 \\
0 & \gamma \lambda_{3}
\end{array}\right] .
\end{aligned}
$$

\section{Numerical stability investigation}

The dynamic stability behavior of the present 2-dof parametrically excited MEMS oscillator is analyzed using two different numerical methods. The first approach described in Section 4.1 is a rather simple one, whereas the second approach described in Section 4.2 is more elegant and makes use of the Floquet theorem. For both stability investigations the system parameters are defined as stated in Table 3 (see [17]). 


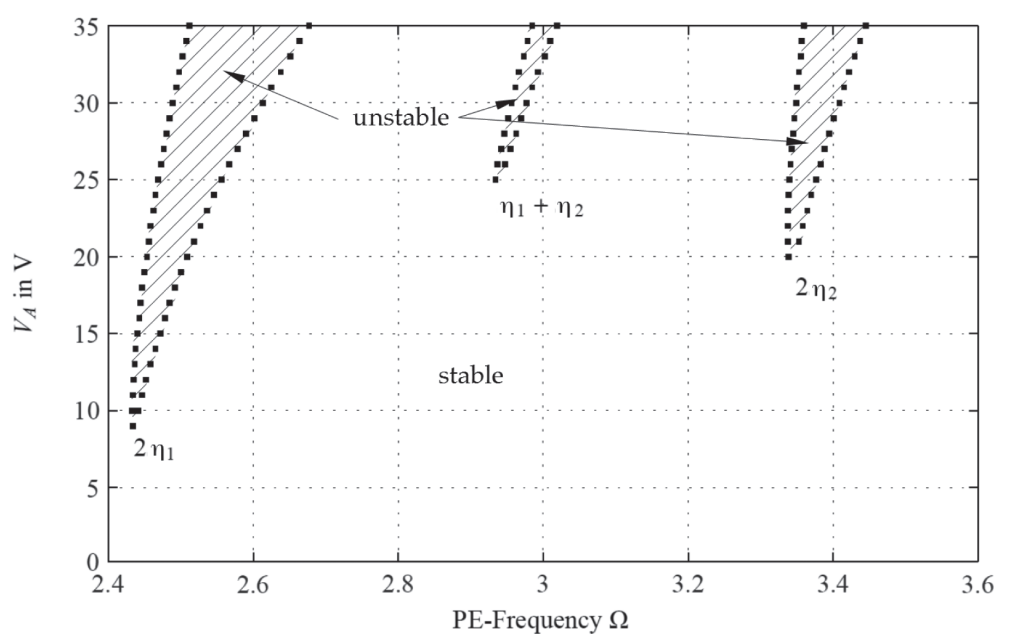

Fig. 4. Stability chart, depicting instability areas due to primary parametric resonances and combination resonances.

\subsection{Numeric integration}

The applied numerical approach is to solve the given equation of motion in the time domain and examine the resulting vibrations in regard of the stability of the system. By using the system parameters stated in Table 3 and the following initial conditions

$$
\begin{aligned}
& z_{1,2}(\tau=0)=\frac{x_{1,2}(t=0)}{x_{0}}=\frac{1 \mu \mathrm{m}}{1 \mu \mathrm{m}}=1, \\
& z_{1,2}^{\prime}(\tau=0)=0
\end{aligned}
$$

Equation (20) is solved for various (parametric excitation) frequencies $\Omega$ of the alternating voltage amplitude $V_{A}$. The stability of the system is analyzed by examining the resulting vibrations after a sufficient time period, which is $\tau=2500$ in terms of the nondimensional time. Figure 4 shows the obtained stability chart with the first parametric regions of instability emerging from

$$
\Omega_{k}^{P R}=\frac{2 \eta_{1}}{k}, \quad k=1,2,3, \ldots
$$

In addition to these primary frequencies, more parametric combination resonances occur at frequency intervals near

$$
\Omega_{k}^{P C R}=\frac{\eta_{1}+\eta_{2}}{k}, \quad k=1,2,3, \ldots
$$

with the same unstable behavior as the primary parametric resonances (see [2]). Comparing these results with the one from the single degree of freedom oscillator described in Section 1, it can be seen that the parametric regions of instability are significantly increased.

Figure 5 shows time series of the systems deflections $z_{1}$ and $z_{2}$ using the primary parametric resonance frequencies obtained from Fig. 4 as excitation frequencies. It can be seen that the oscillation amplitudes increase from the initial values $z_{1,2}=1$ (at $\tau=0$ ), limited only by the present nonlinearities. The behavior of the system, which is reaching a stable limit cycle, is mostly determined by the nonlinearity of the parametric excitation, but the nonlinearities of the springs contribute, too. The linear system becomes unstable at the frequencies $\Omega$ of the parametric excitation mentioned above (see Fig. 6). 

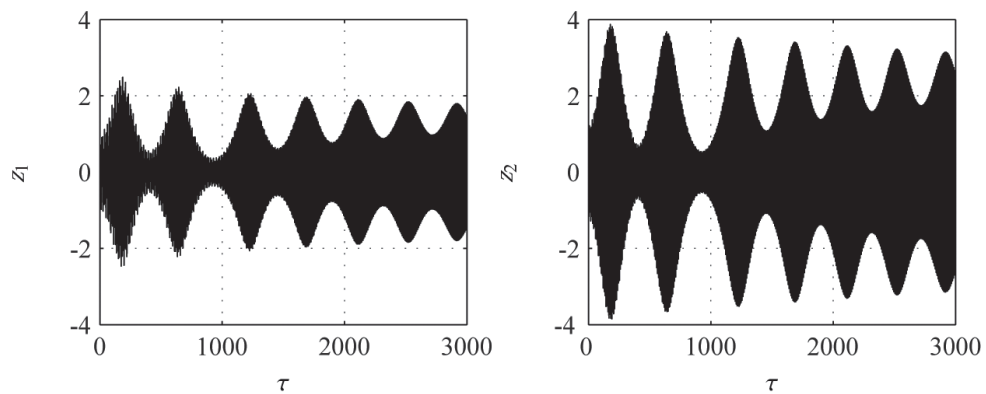

(a) Displacements $z_{1}, z_{2}$ at $\Omega=2 \eta_{1}=2.56$ with $V_{A}=30 \mathrm{~V}$.
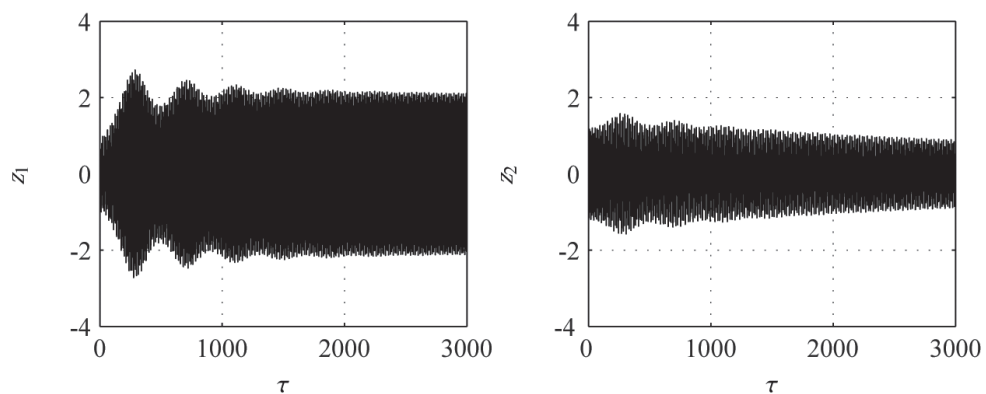

(b) Displacements $z_{1}, z_{2}$ at $\Omega=2 \eta_{2}=3.38$ with $V_{A}=30 \mathrm{~V}$.
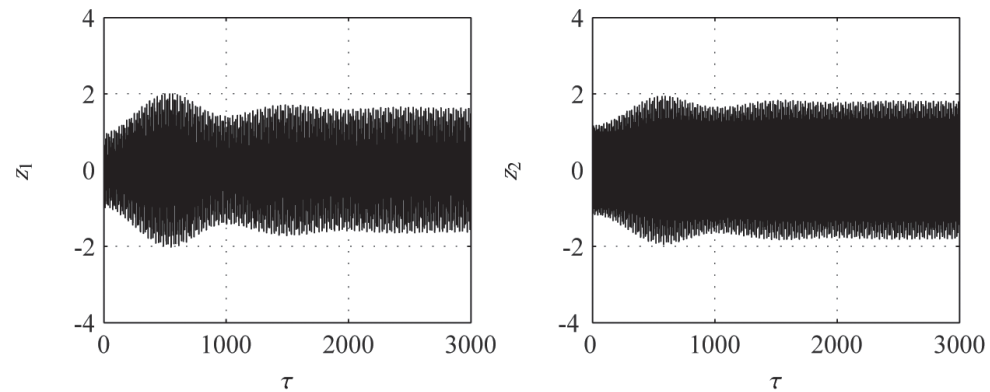

(c) Displacements $z_{1}, z_{2}$ at $\Omega=\eta_{1}+\eta_{2}=2.97$ with $V_{A}=30 \mathrm{~V}$.

Fig. 5. Displacements $z_{1}, z_{2}$ at PE-Resonances $2 \eta_{1}, 2 \eta_{2}$ and $\eta_{1}+\eta_{2}$ with $V_{A}=30 \mathrm{~V}$ and parameters taken from Table 3 .

\subsection{Floquet method}

For analyzes of linear differential equations with time periodic coefficients, the Floquet method (see e.g. [15]) has proven to be very useful. The set of scaled second order differential Eq. (20) can be rewritten as a set of first order differential equations

$$
\mathbf{z}^{\prime}=\mathbf{f}[\mathbf{z}(\tau), \tau] .
$$

The solution of this problem may be written as

$$
\mathbf{z}(\tau)=\mathbf{z}_{0}(\tau)+\Delta \mathbf{z}(\tau),
$$

where $\mathbf{z}_{0}(\tau)$ is the periodic steady state part of the solution and $\Delta \mathbf{z}(\tau)$ is an additional disturbance. The solution $\mathbf{z}(\tau)$ is stable if the following two conditions hold

$$
\mathbf{z}(\tau+T) \leqslant \mathbf{z}(\tau) \Leftrightarrow \Delta \mathbf{z}(\tau+T) \leqslant \Delta \mathbf{z}(\tau) .
$$

The coefficient $T$ is the period of the steady state solution $\mathbf{z}_{0}(\tau)$. By substitution of Eq. (31) into Eq. (30), expanding the expression by a Taylor series for $\mathbf{z}_{0}$ and neglecting nonlinear terms of the disturbance, one obtains

$$
\Delta \mathbf{z}^{\prime}=\mathbf{J} \Delta \mathbf{z}(\tau),
$$



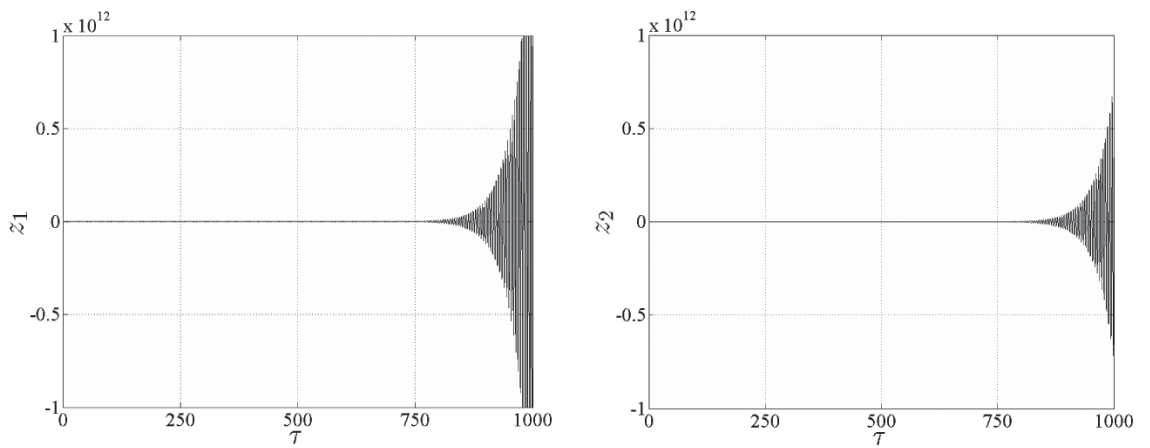

(a) Displacements $z_{1}, z_{2}$ at $\Omega=2 \eta_{1}=2.56$ with $V_{A}=30 \mathrm{~V}$.
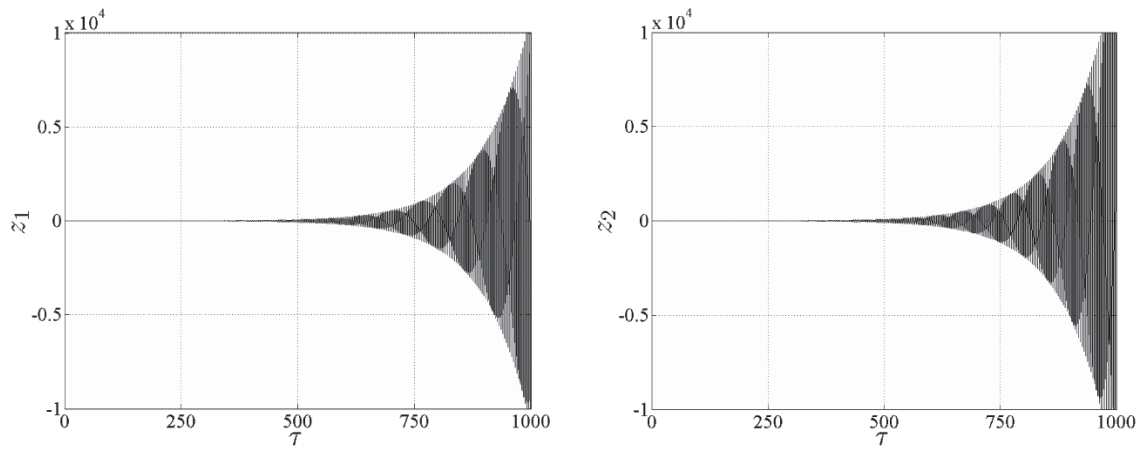

(b) Displacements $z_{1}, z_{2}$ at $\Omega=2 \eta_{2}=3.38$ with $V_{A}=30 \mathrm{~V}$.
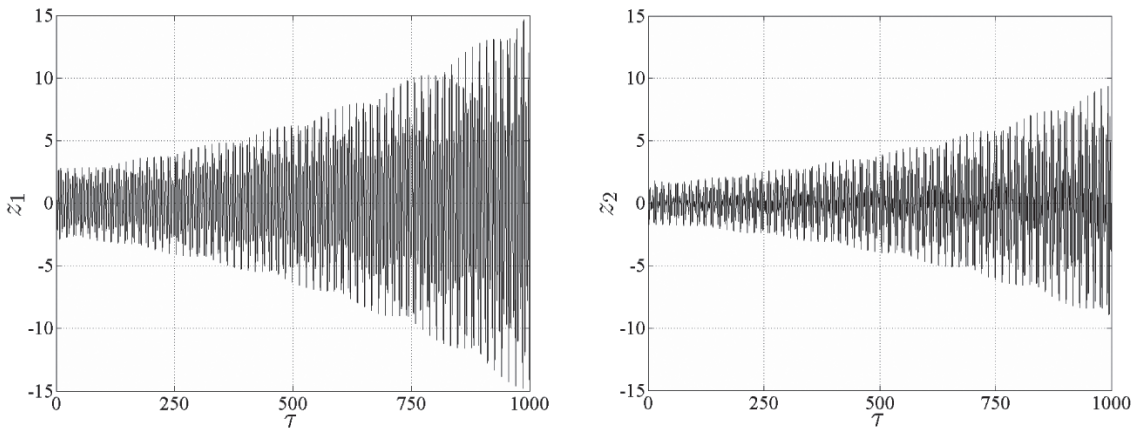

(c) Displacements $z_{1}, z_{2}$ at $\Omega=\eta_{1}+\eta_{2}=2.97$ with $V_{A}=30 \mathrm{~V}$.

Fig. 6. Displacements $z_{1}, z_{2}$ of the linearized system at PE-Resonances $2 \eta_{1}, 2 \eta_{2}$ and $\eta_{1}+\eta_{2}$ with $V_{A}=30 \mathrm{~V}$ and parameters taken from Table 3.

with $\mathbf{J}$ being the Jacobian of Eq. (31). A set of fundamental solutions $\Delta \mathbf{Z}(\tau+T)$ of the disturbance $\Delta \mathbf{Z}$ after one period $T$ can be written as

$$
\Delta \mathbf{Z}(\tau+T)=\Lambda \Delta \mathbf{Z}(\tau),
$$

where $\Delta \mathbf{Z}(\tau)$ is a set of fundamental solutions at the beginning of the period $T$. The so-called Monodromy matrix $\Lambda$ can be calculated by integration of the Jacobian with $n$ independent sets of initial conditions over one period $T$. The coefficient $n$, in this case, represents the number of first order differential equations. In order to determine the stability of the solution $\mathbf{z}(\tau)$, the eigenvalues of the Monodromy matrix $\boldsymbol{\Lambda}$ have to be analyzed. If the magnitude of one of the calculated eigenvalues exceeds one, the system is unstable. If, on the other hand, all eigenvalues are less than one, the system is stable. Figure 7 shows a stability chart of the linearized 2-dof MEMS oscillator for a specific range of the parametric excitation frequency $\Omega$ and the amplitude of the alternating voltage input signal $V_{A}$. 


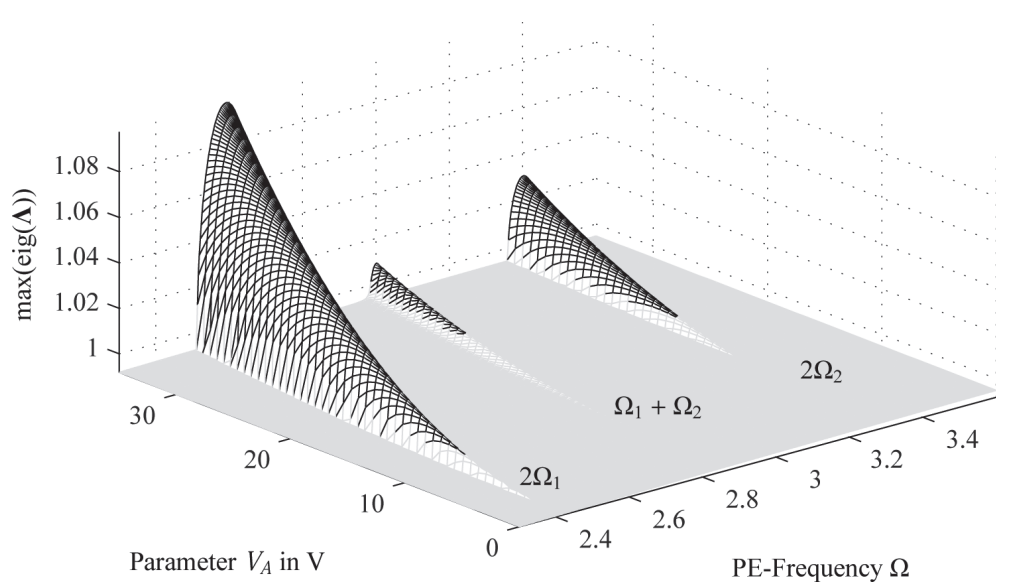

Fig. 7. Stability parameter at primary parametric resonances $\Omega=2 \eta_{1}, \Omega=2 \eta_{2}$ and at the combination resonance $\Omega=\eta_{1}+\eta_{2}$.

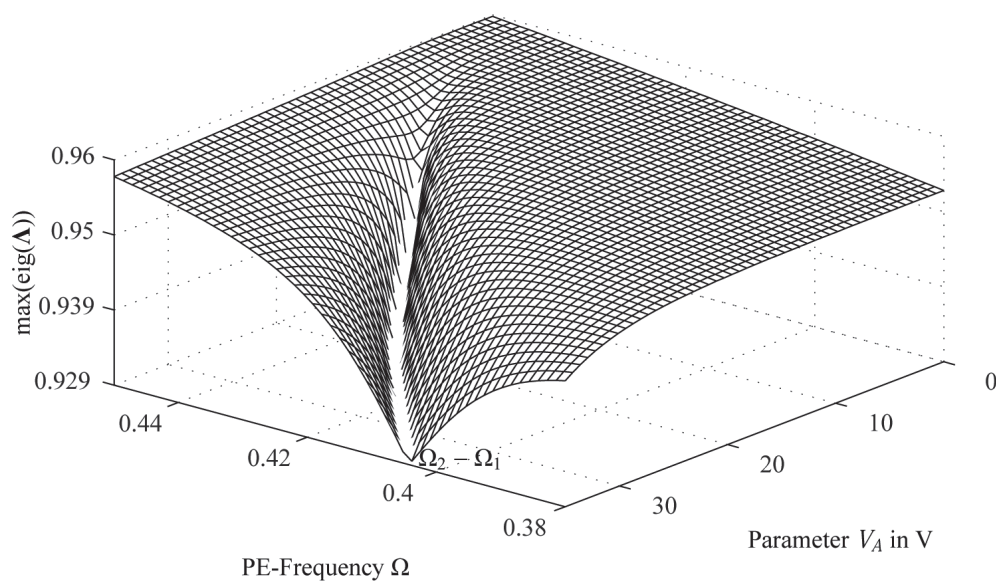

Fig. 8. Stability parameter at the first order parametric anti-resonance $\Omega=\eta_{2}-\eta_{1}$ as a function of the excitation amplitude $V_{A}$.

The stability chart reveals the primary parametric resonances and also the primary combination resonance of the additive type. The areas of instability match reasonably well the ones previously discussed in Section 4.1. Other effects of different nature, for example chaotic behavior, cannot be studied by this method due to its intrinsic limitations. Therefore, intervals of instability where the trivial solution becomes unstable via a Hopf-bifurcation can be detected in the first place, and of course frequency domains with enhanced damping due to the effect of a parametric anti-resonance.

It can be suspected that the fully non-linear 2-dof system may also exhibit chaotic behavior. To find such phenomena, either the numerical method as presented in the previous section, or other suitable methods must be applied. In the course of this study, however, no results where obtained that indicated chaotic behavior. This observation might be based on the fact that the non-linearities in the system are rather small, and that the focus of this work was to investigate only intervals of parametric instabilities and of parametric stabilization.

\section{Parametric anti-resonance}

In addition to the additive parametric combination resonances (see Eq. (29)), so-called parametric anti-resonances (see [13]) exist at subtractive parametric combination frequencies of

$$
\Omega_{k}^{P C R}=\frac{\eta_{2}-\eta_{1}}{k}, \quad k=1,2,3, \ldots
$$



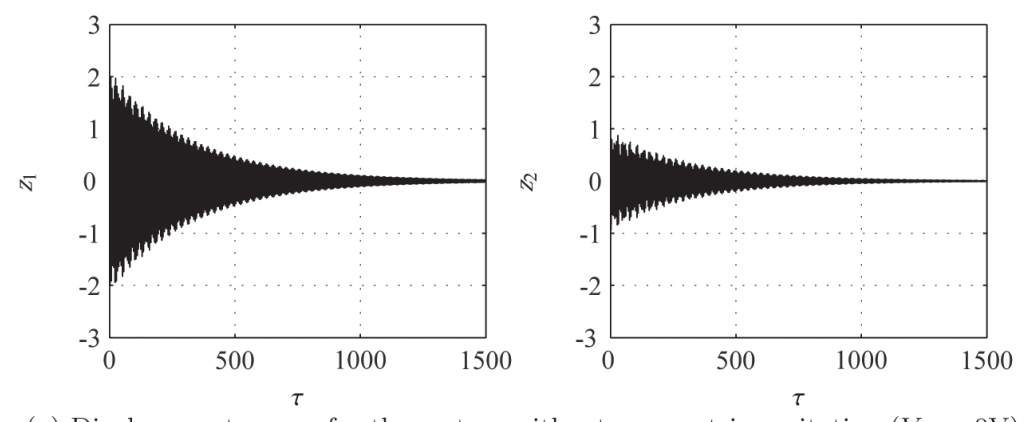

(a) Displacements $z_{1}, z_{2}$ for the system without parametric excitation $\left(V_{A}=0 \mathrm{~V}\right)$.
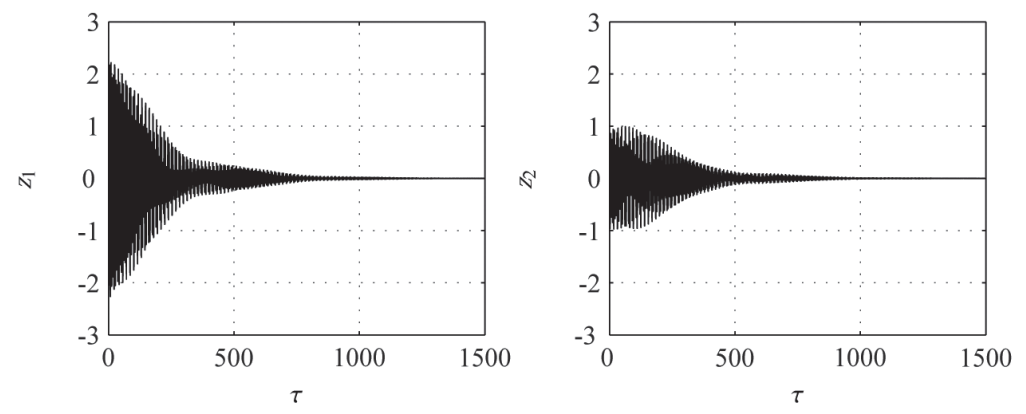

(b) Displacements $z_{1}, z_{2}$ at $\Omega=\eta_{2}-\eta_{1}=0.41$ with $V_{A}=30 \mathrm{~V}$.

Fig. 9. Effect of anti-resonance in the time domain using Eq. (37) as initial conditions.

In contrast to the parametric resonances and additive combination resonances, an anti-resonance has no destabilizing effect on the system. In fact, it enhances the system's damping, see [3,13]. Figure 8 shows the first order antiresonance $\eta_{2}-\eta_{1}$ by using the Floquet method. It can be seen that the eigenvalues of the Monodromy matrix do not exceed 1, but decrease significantly in the region of the anti-resonance.

The positive aspect of the anti-resonance phenomenon can be examined best by comparing vibration signals in the time domain. Figure 9 shows time series, using initial conditions as defined by Eq. (37), which are close to the limit cycles of the vibration amplitudes as shown in Fig. 5(b). The system's deflections are analyzed for the system without parametric excitation $\left(V_{A}=0\right)$ and for the parametric anti-resonance frequency $\Omega=\eta_{2}-\eta_{1}=0.41$.

$$
z_{1}(\tau=0)=2, \quad z_{2}(\tau=0)=1, \quad z_{1,2}^{\prime}(\tau=0)=0 .
$$

While Fig. 9(a) basically shows the expected exponential decay of the vibration amplitudes, a quite different behavior can be seen in the time series below. Due to the parametric excitation at $\Omega=\eta_{2}-\eta_{1}=0.41$ with a PE-amplitude of $V_{A}=30 \mathrm{~V}$, a rather fast decay of the vibration signal $z_{1}$ occurs. At $\tau \simeq 300$ the vibration amplitudes of $z_{1}$ are less than half of those for the system without parametric excitation. Initially, this rapid decay does not occur for the vibration signal $z_{2}$ at the other mass $m_{2}$. In fact, first one encounters a modulated vibration signal and only after 100 time units a reduction of the vibration level starts. However, at $\tau=500$ also for $z_{2}$ the signal is less than half of that for the system without PE at the anti-resonance frequency. The enhanced vibration decay is remarkable, but still not the maximum effect that may be achieved for a system optimized in regard of the anti-resonance effect.

When looking at these simulation results, one might think that parametric excitation acts as an additional damping mechanism for the system. In fact, an energy transfer is initiated by PE between the first and the second vibrational mode, see [4]. Vibrational energy that is concentrated in one vibrational mode is gradually moved to the other mode. This energy transfer between two modes goes on and on as long as PE is active and energy is still present. By this mechanism advantage is taken of the fact that in engineering systems higher modes have a higher damping ratio. Therefore, energy can dissipate faster compared to the conventional system without PE, if energy is moved to a mode of higher order. Since the energy transfer is not unidirectional, modulated vibrations are observed and for an optimal effect, the system design may be optimized accordingly. 


\section{Conclusion}

Single degree of freedom MEMS oscillators exhibit parametric resonances at frequency intervals of $2 \eta_{1} / k$. Due to the presence of damping the resonances of higher order $(k>1)$ are not relevant for application purpose. Electronic filtering is a possible application of such 1-dof MEMS. Because of the distinctive different stability behavior in the stable and the unstable parameter domain, almost ideal filter characteristics can be achieved (see [10]).

In the second part this study explores advantages that can be gained from a novel 2-dof MEMS design. The use of a two degree of freedom MEMS oscillator, as investigated here, is motivated by two major advantages compared to a single degree of freedom design, particularly concerning a band-pass filter application. Not only are the usable regions of parametric instability increased (see Eq. (29)), but also a very advantageous anti-resonance phenomenon occurs. This phenomenon may be used to improve the damping behavior of the system in order to further increase the quality of a band-pass filter design. Concerning a parametric MEMS mass sensor, the anti-resonance phenomenon might be beneficial for the increase of the sampling time. If the system transitions between an unstable and a stable state, the enhanced damping causes the oscillator to reach a static condition even faster (see Fig. 9).

\section{Acknowledgments}

The authors would like to thank Prof. Dr. Ulrich Schmid, Vienna Univ. of Technology, for the fruitful discussions on the design of MEMS.

\section{References}

[1] K.L. Turner, S.W. Shaw, B.E. DeMartini, J.F. Rhoads and J. Moehlis, Linear and nonlinear tuning of parametrically excited mems oscillators, Journal of Microelectromechanical Systems 16 (2007), 310-318.

[2] M. Cartmell, Introduction to Linear, Parametric and Nonlinear Vibrations, Chapman and Hall, London, 1990.

[3] H. Ecker, Suppression of Self-excited Vibrations in Mechanical Systems by Parametric Stiffness Excitation, Argesim/ASIM, Vienna, 2005.

[4] T. Pumhoessel and H. Ecker, Vibration suppression and energy transfer by parametric excitation in drive systems, Proceedings of the Institution of Mechanical Engineers, Part C: Journal of Mechanical Engineering Science 226(8) (2012), 2000-2014.

[5] B.T. Khuri-Yakub, J.P. Raskin, A.R. Brown and G.M. Rebeiz, A novel parametric-effect mems amplifier, Journal of Microelectromechanical Systems 9 (2000), 528-537.

[6] O.O. Awadelkarim, J.W. Gardner and V.K. Varadan, Microsensors, MEMS, and Smart Devices, John Wiley and Sons, Chicester, 2001.

[7] P.G. Hartwell, N.C. MacDonald, S.H. Strogatz, K.L. Turner, S.A. Miller and S.G. Adams, Five parametric resonances in a microelectromechanical system, Nature 396 (1998), 149-152.

[8] J.M. Madou, Fundamentals of Microfabrication and Nanotechnology, Francis and Taylor, Boca Raton, 2012.

[9] J. Rhoads, S.W. Shaw, K. Turner and R. Baskaran, Tunable microelectromechanical filters that exploit parametric resonance, Journal of Vibration and Acoustics 127 (2005), 423-432.

[10] J. Rhoads, S.W. Shaw, K. Turner and R. Baskaran, Generalized parametric resonance in electrostatically actuated microelectromechanical oscillators, Journal of Sound and Vibration 296 (2006), 797-829.

[11] K.A. Shaw, S.G. Adams, F.M. Bertsch and N.C. MacDonald, Independent tuning of linear and nonlinear stiffness coefficients, Journal of Microelectromechanical Systems 7 (1998), 172-180.

[12] J.F. Rhoads, S.W. Shaw, K.L. Turner and R. Baskaran, Parametrically excited mems-based filters, Proceedings of the IUTAM Symposium on Chaotic Dynamics and Control of Systems and Processes, 2003, pp. 137-146.

[13] A. Tondl, To the problem of quenching self-excited vibrations, Acta Technica CSAV 43 (1998), 109-116.

[14] K.L. Turner and W. Zhang, Design and analysis of a dynamic mem chemical sensor, Proceedings of the American Control Conference, 2001, pp. 1214-1218.

[15] F. Verhulst, Nonlinear differential equations and dynamical systems, Springer, Berlin, 2006.

[16] R. Baskaran, W. Zhang and K.L. Turner, Effect of cubic nonlinearity on auto-parametrically amplified resonant mems mass sensor, Sensors and Actuators A 102 (2002), 139-150.

[17] J. Welte, Parametric Excitation in Micro-Electro-Mechanical Systems, Master Thesis, TU-Vienna, Vienna, 2012.

[18] A. Zielke, C.B. Burgner, Z. Yie and K.L. Turner, Comparison of parametric and linear mass detection in the presence of detection noise, Journal of Micromechanics and Microengineering 21 (2011), 1-5.

[19] W. Zhang, R. Baskaran and K. Turner, Effect of cubic nonlinearity on auto-parametrically amplified resonant mems mass sensor, Sensors and Actuators A: Physical 102 (2002), 139-150.

[20] W. Zhang and K.L. Turner, A mass sensor based on parametric resonance, Solid-State Sensor, Actuator and Microsystems Workshop, 2004, pp. 49-52. 

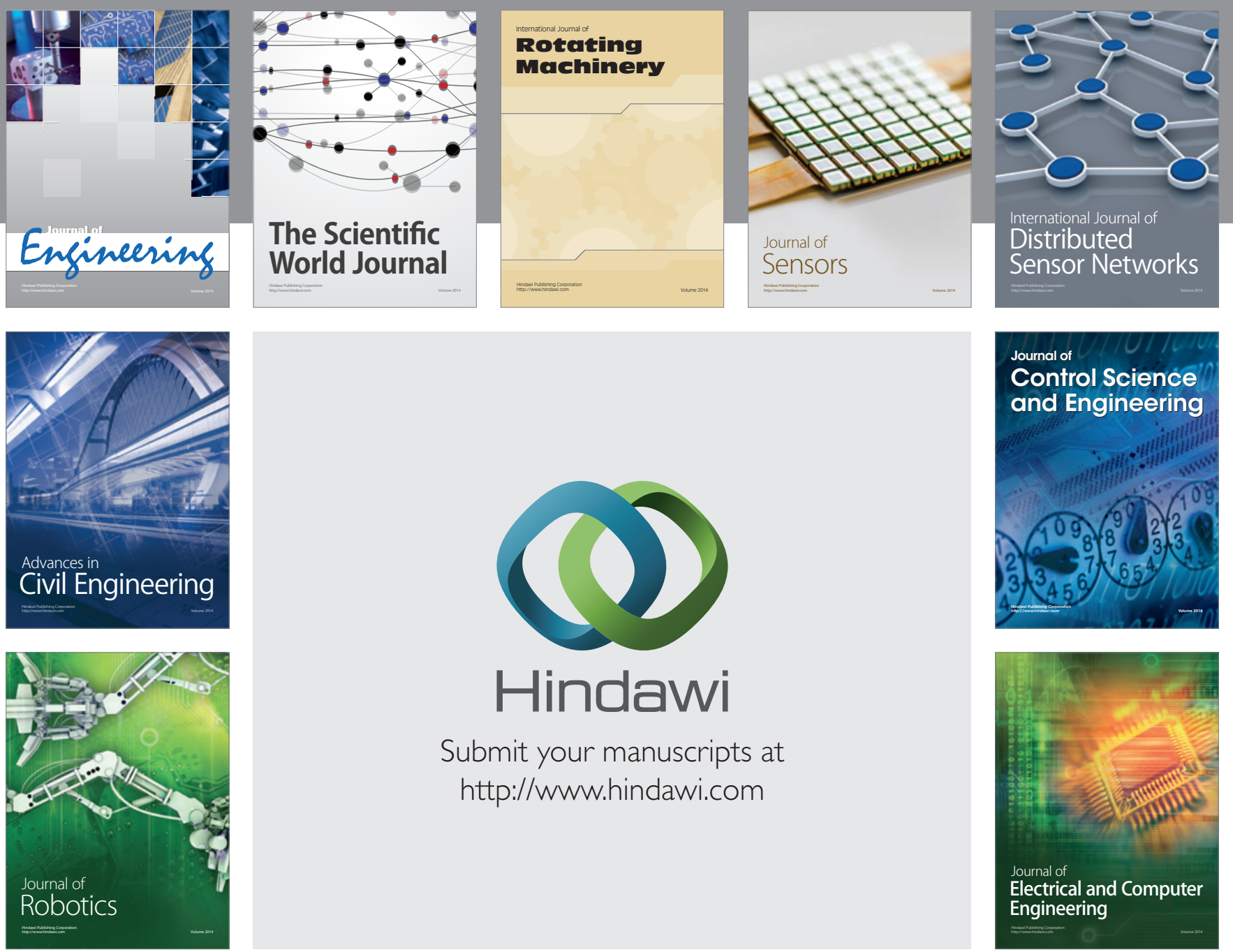

Submit your manuscripts at

http://www.hindawi.com
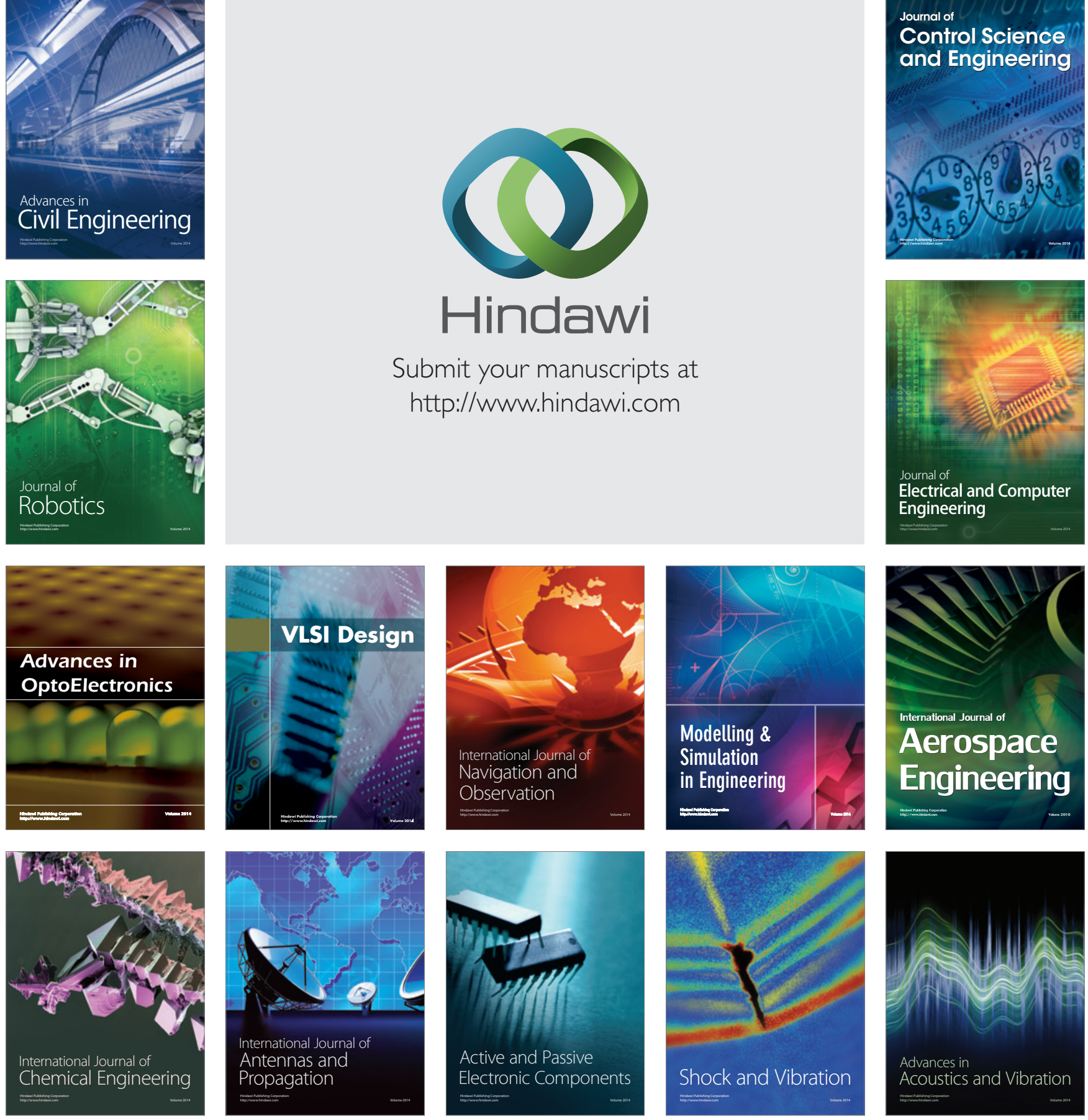